\title{
OCORRÊNCIA DE OOCISTOS DE Cryptosporidium spp. EM RÃS-TOURO (Lithobates catesbeianus SHAW, 1802), NO MUNICÍPIO DE UBERLÂNDIA, MG, BRASIL
}

\author{
Aline Diniz Cabral, ${ }^{1}$ Marcio Hipolito, ${ }^{2}$ Dagmar Diniz Cabral ${ }^{3}$ e Noé Ribeiro da Silva ${ }^{4}$ \\ 1. Instituto Biológico. G-mail: alinedica@netsite.com.br \\ 2. Instituto Biologico \\ 3. Universidade Federal de Uberlândia \\ 4. Universidade Federal de Uberlândia.
}

\section{RESUMO}

O presente trabalho teve como objetivo detectar a ocorrência de oocistos de Cryptosporidium spp. em raspagens da mucosa intestinal e amostras de fezes de rãs-touro (Lithobates catesbeianus, Shaw, 1802) em confinamento, na fazenda escola da Universidade Federal de Uberlândia, MG, utilizando a técnica de coloração de Ziehl Neelsen modificada. Em 28 amostras fecais, 14,28\% (4/28) foram positivas para Cryptosporidium spp., sendo 7,14\% positivas em fezes de rãs com menos de três meses e 7,14\% positivas em fezes de rãs com mais de três meses. Em dezesseis amostras de raspagem intestinal, $75 \%(10 / 16)$ foram positivas, sendo $50 \%$ em girinos e $25 \%$ em rãs adultas. Esta pesquisa permite identificar a situação e recomendar medidas de controle, visando a prevenir ou a retardar o aparecimento dessa parasitose nos demais animais, visto que trata-se de uma zoonose importante em saúde pública.

PALAVRAS-CHAVES: Cryptosporidium spp, protozoário, rã-touro, Lithobates catesbeianus.

\section{ABSTRACT}

OCCURRENCE OF Cryptosporidium spp. OOCYSTS IN BULLFROG (Lithobates catesbeianus SHAW, 1802)

The objective of the present study was to detect the occurrence of Cryptosporidium spp. oocysts in intestinal mucosa scraping and feces samples from bullfrogs (Lithobates catesbeianus Shaw, 1802) confined at the school farm of Federal University of Uberlândia, MG, Brazil. The modified Ziehl Neelsen staining technique was used. Out of 28 fecal samples, 14.28\% (4/28) were positive for Cryptosporidium spp., with $7.14 \%$ positive samples in feces of frogs under 3 months of age and $7.14 \%$ in feces of frogs over 3 months of age. Form the 16 intestinal scraping samples, $75 \%$ (10/16) were positive, $50 \%$ in tadpoles and $25 \%$ in adult frogs. This research allows the situation identification and the recommendation of measures to control this parasite, aiming at preventing or delaying the appearance of this parasitism in other animals, since it an important zoonosis to public health.

KEYWORDS: Cryptosporidium spp, protozoan, bullfrog, Lithobates catesbeianus.

\section{INTRODUÇÃO}

Cryptosporidium são protozoários parasitas de distribuição mundial, cujas principais vias de transmissão ocorrem mediante a ingestão de água e alimentos contaminados por oocistos esporulados. A infecção por coccídios intestinais provoca alterações na mucosa do intestino delgado, atrofia das vilosidades e hiperplasia da cripta, resultando em síndrome da má-absorção (THOMPSON \& CHALMERS, 2002). 
O diagnóstico dessa protozoose pode ser realizado pela observação das formas endógenas do parasito em cortes histológicos do intestino do hospedeiro, facilmente identificado com a coloração hematoxilina-eosina ou pela demonstração dos oocistos presentes nas fezes, mediante o emprego de técnicas de coloração como a de Ziehl Neelsen modificada (HENRIKSEN \& POHLENZ, 1981; FAYER, 1997). Outros métodos de diagnósticos já foram descritos para detecção de oocistos nas fezes, utilizando técnicas imunológicas como a imunofluorescência direta (SMITH et al., 2007), ensaio imunoabsorvente ligado à enzima e imunocromatografia (JOHNSTON et al., 2003), com base na reação em cadeia pela polimerase (WIDMER et al., 2002). Os métodos sensíveis e específicos são poucos utilizados em análises rotineiras, pois requerem competências técnicas e equipamentos sofisticados.

Assim como os demais coccídios, os oocistos de Cryptosporidium são extremamente resistentes à maioria dos desinfetantes empregados, como hipoclorito, hidróxido de sódio, fenóis e cresóis. Entretanto, são suscetíveis à dessecação e às altas temperaturas. Dessa forma, para se prevenir a infecção, medidas de higiene e sanitação - incluindo limpeza, desinfecção e boas condições de ventilação e insolação são - especialmente recomendadas nos locais de alojamento dos animais jovens (FAYER, 1997).

São raros os relatos de ocorrências de Cryptosporidium em anfíbios, tanto silvestres como mantidos em laboratórios (GREEN el al., 2003; JIRKU et al., 2008), sendo que em animais de criação comercial não há citação alguma.

No Brasil, é criada a rã-touro americana, antes denominada Rana catesbeiana SHAW, 1802, e recentemente renomeada como Lithobates catesbeianus (FROST et al., 2006). O país tem uma situação privilegiada quanto à criação comercial de rãs: o mercado potencial interno é maior que a oferta e há demanda espontânea por esse tipo de carne; no mercado internacional, também há mais demanda do que oferta. Segundo CRIBB (2009), a produção brasileira de carne de rã tem crescido continuamente: de dez para 639 toneladas no período de 1986 a 2006. Enquanto o crescimento mundial foi de $1.296 \%$, o do Brasil alcançou $6.290 \%$. O país é o maior produtor das Américas, tendo obtido uma participação, entre 2000 e 2006 , de $58 \%$ a $68 \%$ do total produzido no continente. Segundo FERREIRA
(2009), a média de produção nacional é de quatrocentas toneladas por ano, absorvidas no mercado interno. A carne de rã é um produto apreciado pelos consumidores por apresentar baixo teor de gordura, em média 1,5\%, condizente com a tendência contemporânea de busca por alimentação saudável.

A ranicultura se tornou uma das atividades em ascensão no Brasil e no mundo com o aumento do poder consumidor da classe média alta, que expressou o desejo de obter outras fontes de proteína animal que não as convencionais. Ressalte-se que o Brasil detém a melhor tecnologia de produção de rã-touro em cativeiro e é também um dos maiores produtores mundiais. Até o momento não se tem registro no país da existência de levantamentos sobre a infecção por Cryptosporidium em anfíbios e em particular, na rã-touro.

Os cuidados sanitários e a pesquisa sobre agentes agressores ou potencialmente patogênicos para a rã-touro se justificam devido ao avançado conhecimento zootécnico já alcançado na criação dessa espécie. Considerando-se a produção brasileira entre 400 e $600 \mathrm{~kg}$ anuais (CRIBB, 2009; FERREIRA, 2009), com exportação para vários países, inclusive de animais vivos, o processo de criação e venda deve ser cercado de responsabilidade e conhecimento de potencias agentes patogênicos e medidas de controle. As medidas visam a garantir melhor qualidade do produto final, seja carne, couro ou anaimal vivo para exportação. O país importador pode reconhecer um risco para suas espécies nativas. No entanto, segundo XIAO et al. (2004), não há relação entre anfíbios e Cryptosporidium em saúde pública.

Este trabalho visa a contribuir para suprir uma lacuna deixado pela falta de estudos da presença de protozoários em anfíbios no Brasil, incluindo-se a rã-touro, com poucas citações. São relatados somente Giardia agilis, Entamoeba sp, Trichomonas sp, Hexamita sp e Karotomorfa sp, de acordo com BUENO-GUIMARÃES (1999) e HIPOLITO \& SOUZA JR. (2001), sendo praticamente inexistentes estudos em nosso meio sobre esses parasitos (HIPOLITO \& $\mathrm{BACH}, 2002)$.

Aqui, objetivou-se detectar a ocorrência do protozoário Cryptosporidium em fezes e em raspado de mucosa do intestino delgado de rã-touro (Lithobates catesbeianus), girinos e adultos, do ranário experimental da Universidade Federal de Uberlândia, MG. 


\section{MATERIAL E MÉTODOS}

A pesquisa foi realizada no ranário experimental existente na fazenda da Universidade Federal de Uberlândia, MG. Coletaram-se fezes de rãs de até três meses de idade (quatorze amostras) e de rãs acima de três meses (quatorze amostras), em piscinas das baias de engorda, devido ao hábito das rãs de defecarem quase exclusivamente na água. Um pool de fezes foi coletado em cada baia pela manhã (duas vezes por semana), armazenando-se o material em sacos plásticos devidamente identificados, que posteriormente foram transportados até o Laboratório de Parasitologia da Universidade Federal de Uberlândia, para processamento.

Também foram utilizados oito girinos e oito rãs adultas (acima de três meses de idade) para coleta dos intestinos e posteriores raspagens da mucosa do intestino delgado. Os animais foram recolhidos vivos pela manhã (uma vez por semana), acondicionados em sacos plásticos, identificados e encaminhados ao laboratório. Eles foram eutanasiados (por atordoamento e decapitação) - segundo a Resolução $n^{0} 714$, de 20 de junho de 2002, do Conselho Federal de Medicina Veterinária - para a retirada das amostras de intestino, que foram fixadas em formalina a $10 \%$, para aguardar o processamento.
Para a concentração dos oocistos empregou-se a técnica de centrífugo-sedimentação pelo formaldeído-éter e em seguida foram confeccionados esfregaços fecais. No caso das amostras intestinais, confeccionaram-se lâminas com raspado da mucosa do intestino delgado com auxílio de bisturi estéril. Após secarem em temperatura ambiente por quatro horas, as lâminas foram coradas a quente pela técnica de Ziehl-Neelsen modificada, de HENRIKSEN \& POHLENZ (1981), e descoloradas por imersão em solução álcool-ácido a $3 \%$. As lâminas foram examinas em microscópio em aumento de 400x e 1.000x até a observação do oocisto do Cryptosporidium spp. Para verificar as possíveis associações entre as diferentes variáveis estudadas e as proporções comparativas das distintas categorias, realizou-se o teste de $\mathrm{Z}$ com o nível de significância estabelecido em $5 \%$.

\section{RESULTADOS E DISCUSSÃO}

Observaram-se oocistos de Cryptosporidium spp. em 14,3\% (4/28) das amostras fecais, sendo 7,14\% positivas em fezes de rãs com menos de três meses e 7,14\% positivas em fezes de rãs com mais de três meses. Em dezesseis amostras de raspagem intestinal, $62,5 \%$ (10/16) foram positivas, sendo $50 \%$ em girinos e $25 \%$ em rãs adultas (Tabela 1 ).

TABELA 1. Ocorrência de Cryptosporidium spp. em rãs-touro criadas em sistema de confinamento no ranário experimental da fazenda da Universidade Federal de Uberlândia, MG, 2005

\begin{tabular}{|c|c|c|c|c|}
\hline Animais em estudo & Amostras & $\begin{array}{l}\text { Positivas } \\
\text { (N) }\end{array}$ & Negativas & $\begin{array}{c}\text { Representatividade dos } \\
\text { positivos (n) }\end{array}$ \\
\hline Fezes de rãs com mais de três meses & $14(\mathrm{n})$ & $2(7,15 \%)$ & $12(42,85 \%)$ & $14,28 \%$ \\
\hline Fezes de rãs com menos de três meses & $14(\mathrm{n})$ & $2(7,15 \%)$ & $12(42,85 \%)$ & $14,28 \%$ \\
\hline Total & $28(\mathrm{~N})$ & $4(14,3 \%)$ & $24(85,7 \%)$ & - \\
\hline Raspagem intestinal de girinos & $8(\mathrm{n})$ & $8(50 \%)$ & $0(0 \%)$ & $100 \%$ \\
\hline Raspagem intestinal de rãs adultas & $8(n)$ & $4(25 \%)$ & $4(25 \%)$ & $50 \%$ \\
\hline Total & $16(\mathrm{~N})$ & $10(62,5 \%)$ & $4(25 \%)$ & - \\
\hline
\end{tabular}

\section{DISCUSSÃO}

Pelo menos 22 espécies do gênero Cryptosporidium foram identificadas por diferenças na ocorrência e preferência pelo hospedeiro, morfologia e sítio de infecção, sendo vinte espécies aceitas, incluindo peixes, anfíbios, répteis, aves e mamíferos (FAYER, 2010). Em 2008, quatro novas espécies de Cryptosporidium foram descritas, com base em características biológicas, morfológicas e moleculares. RYAN et al. (2008) isolaram C. fayeri de fezes de cangurus vermelhos (Macropus rufus); POWER \& RYAN (2008), C. macropodum de 
fezes de cangurus cinza (Macropus giganteus); JIRKU et al. (2008), C. fragile do estômago de sapos black-spined (Duttaphrynus melanostictus); e FAYER et al. (2008), C. ryanae de fezes de bovinos (Bos taurus).

Sabe-se que os peixes, anfíbios e répteis são hospedeiros do oocisto de Cryptosporidium, mas a sua capacidade infectante para os mamíferos ainda permanece desconhecida, como demonstra CRANFIEL \& GRACZYK (1996); porém, anfíbios devem ser considerados como vetores de potencial mecânico, que podem aumentar a transmissão de Cryptosporidium serpentis entre répteis e centros herpetológicos (GRACZYK et al., 1998). Cryptosporidium parvum induzido em aves debilitadas por infecções respiratórias (LINDSAY et al., 1987) não é infectante em peixes, anfíbios e répteis (GRACZYK et al., 1996).

Esse parasito tem sido documentado na mucosa intestinal de anfíbios (O`DONOGHUE, 1995). Os estágios de Cryptosporidium parvum em outras espécies constituem o primeiro relato de criptosporidiose respiratória confirmada por cortes histológicos, mostrando trofozoítos, gamontes e oocistos em anfíbios e répteis (GRACZYK et al., 1996).

Poucos relatos de Cryptosporidium em anfíbios foram pesquisados em alguns países, sendo que encontrou-se o oocisto nas fezes de rãs nativas, segundo CRAWSHAW \& MEHENEN (1987); no sapo comum americano (Bufo americanus), conforme CRANFIEL \& GRACZYK (1996); e, recentemente, no estômago de anuros da família Bufonidae (Duttaphrynus melanostictus), caracterizando a nova espécie, Cryptosporidium fragile (JIRKU et al., 2008). No que se refere aos reservatórios e portadores, estudos recentes estão descobrindo novas espécies de animais suscetíveis a esse protozoário, sendo possível a detecção de Cryptosporidium spp. em todos os tipos de vertebrados: animais domésticos, animais selvagens, aves, répteis, anfíbios e peixes.

Segundo as análises estatísticas, usando o teste de $Z$, observou-se diferença significativa $(p<0,05)$ ao se compararem os achados nos raspados de mucosa intestinal de girinos com os achados nos raspados de mucosa intestinal das rãs adultas. Todos os girinos apresentaram positividade para o Cryptosporidium, ou seja, as oito amostras de raspagem intestinal dos girinos foram positivas. Esse resultado pode ser devido à origem e à qualidade da água de abastecimento dos tanques de girinagem. A água é oriunda das pastagens de bovinos e, sendo os girinos exclusivamente aquáticos, podem estar adquirindo o parasito por meio da água contaminada pelas fezes dos bovinos.

\section{CONCLUSÃO}

Esta pesquisa representa um avanço no estudo do protozoário Cryptosporidium, pois nela foram utilizadas somente rãs-touro, diversificando-se as faixas etárias, verificando-se a ocorrência de oocistos em todas elas e, em menor grau, nas metamorfoseadas (adultas). Com base no estudo, propõem-se medidas profiláticas na água de abastecimento dos tanques de girinagem oriunda das pastagens de bovinos, assim como a realização de análises laboratoriais periódicas, a fim de controlar a ocorrência e a disseminação dessa parasitose, evitando perdas econômicas. Outras medidas de controle e prevenção seriam a instituição da quarentena, no caso de introdução de novos animais no ranário, e a adoção de medidas de higiene e sanitação, incluindo limpeza, desinfecção e boas condições de ventilação e insolação nas baias das rãs.

Devido à possibilidade de transmissão ao homem, dependendo da espécie de Cryptosporidium, o manejo desses animais pela equipe técnica e pelos tratadores pode representar risco de infecção humana. Dessa forma, torna-se imprescindível a realização de pesquisas para melhor esclarecimento sobre a epidemiologia e caracterização molecular desse parasito em rãs-touro. Ressalta-se que este foi um trabalho de iniciação científica (Fapemig), que detectou apenas a ocorrência do protozoário Cryptosporidium spp. (Figura 1).

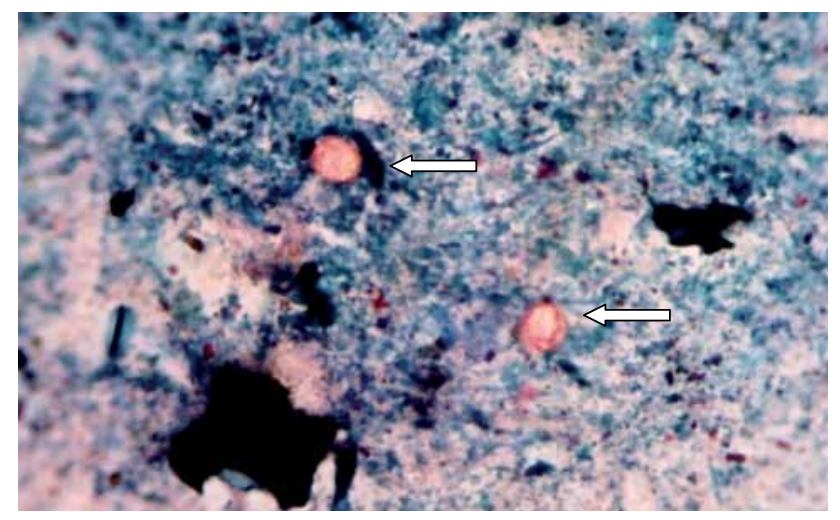

FIGURA 1. Fotografia de Cryptosporidium spp. em rãs-touro do ranário experimental da Universidade Federal de Uberlândia, MG, em aumento de $100 x$. 


\section{REFERÊNCIAS}

ANGUS, K. W. Cryptosporidiosis in man, domestic animals and birds: a review. Journal of Royal Society of Medicine, v. 76, p. 62-70, 1983.

BUENO-GUIMARÃES, H. M. Avaliação da resposta da Rana catesbeiana frente às variações ambientais: determinação das condições ideais de manutenção em biotério e da resposta aos poluentes aquáticos. 1999. 180 f. [Dissertação (Mestrado) - Faculdade de Medicina, Universidade de São Paulo, São Paulo. Disponível em: <http://www.teses.usp.br/>

CRANFIEL, M. R.; GRACZYK, T. K. Cryptosporidiosis. In: MADER, D. R. (Ed.). Manual of reptile medicine and surgery. Philadelphia: W. B. Saunders Company, 1996. p. 359-364.

CRANFIEL, M. R.; GRACZYK, T. K. Experimental infection of elaphid snakes with Cryptosporidium serpentis (Apicomplexa: Cryptosporidiidae). Journal of Parasitology, v. 80, p. 823-826, 1994.

CRAWSHAW, G. L.; MEHREN, K. G. Cryprosporidiosis in zoo animals. In: IPPEN, R.; SCHROEDER, H. D. (Eds.). Erkrankungen der zootiere. Berlin: Academie Verlag, 1987. p. 383-393.

CRIBB, A. Y. Embrapa inicia projeto para fortalecer a ranicultura na região Sudeste. Disponível em: $<\mathrm{http}: / / \mathrm{www}$. clicnews.com.br/tecnologia/view.htm?id=93557>. Acesso em: 10 abr. 2009.

FAYER, R. Cryptosporidium and criptosporidiosis. Boca Raton: CRC Press, 1997. 251 p.

FAYER, R. Taxonomy and species delimitation in Cryptosporidium. Experimental Parasitology, v. 124, p. 90-97, 2010.

FAYER, R.; SANTIN, M.; TROUT, J. M. Cryptosporidium ryanae n. sp. (Apicomplexa: Cryptosporidiidae) in cattle (Bos taurus). Veterinary Parasitology, v. 156, p. 191-198, 2008.

FERREIRA, C. M. Ranicultura: uma alternativa para um bom empreendimento. Disponível em: $<$ http://www.criareplantar.com. br/noticia/ler/?idNoticia=500>. Acesso em: 10 abr. 2009 .

FROST, D. R.; GRANT, T.; FAIVOVICH, J.; BAIN, R. H.; HAAS, A.; HADDAD, C. F. B.; DE AS, R.; CHANNING, A.; WILKINSON, M.; DONNELLAN, S. C.; RAXWORTHY, C. J.; CAMPBELL, J. A.; BLOTTO, B. L.; MOLER, P.; DREWES, R. C.; NUSSBAUM, R. A.; LYNCH, J. D.; GREEN, D. M.; WHEELER, W. C. The amphibian tree of life. Bulletim of American Museum of Natural History, v. 297, p.1-370, 2006.

GOODWIN, M. A. Cryptosporidioses in birds-a review. Avian Pathology, v. 18, p. 368-384, 1989.
GRACZYK, T. K. Fish, Amphibians and Reptiles. In: FAYER, R.; XIAO, L. (Eds.). Cryptosporidium and Cryptosporidiosis. Boca Raton: IWA Publishing, 2008. p. 387-394.

GRACZYK, T. K.; FAYER, R.; CRANFIEL, M. R. Cryptosporidium parvum is not transmissible to fish, anphibians, or reptiles. Journal of Parasitology, v. 82, n. 5, p. 748-751, 1996.

GRACZYK, T. K.; CRANFIEL, M. R.; GEITNER, M. E. A. Multiple Cryptosporidium serpentis oocyst isolates from captive snakes are not transmissible to amphibians. Journal of Parasitology, v. 84, n. 6, p. 1.238-1.300, 1998.

GREEN, S. L.; BOULEY, D. M.; JOSLING, C. A.; FAYER, R. Cryptosporidiosis associated with emaciation and proliferative gastritis in a laboratory south African clawed frog. Advances in Veterinary Science and Comparative Medicine, v. 53, p. 81-84, 2003.

HIPOLITO, M.; BACH E. E. Patologias em rã-touro (Rana catesbeiana SHAW, 1802): primeira revisão da bibliografia brasileira. Arquivos do Instituto Biológico, v. 69, n. 2, p. 113-120, 2002.

HIPOLITO, M.; SOUZA JR. F. L. Anfíbios: uma sinopse de suas doenças. In: ENCONTRO NACIONAL DE RANICULTURA, 11., 2001, Bragança Paulista. Cursos-Anais... Bragança Paulista: Aberta/Ranário Beija-Flor, 2001. p. 1-34.

JIRKU, M.; VALIGUROVÁ, A.; KOUDELA, B.; KRIZEK, J.; MODRÝ, D.; SLAPETA, J. New species of Cryptosporidium Tyzzer, 1907 (Apicomplexa) from amphibian host: morphology, biology and phylogeny. Folia Parasitologica, v. 55, n. 2, p. 8184, 2008.

JOHNSTON, S. P.; BALLARD, M. M.; BEACH, M. J.; CAUSER, L.; WILKINS, P. P. Evaluation of three commercial assays for detection of Giardia and Cryptosporidium organisms in fecal specimens. Journal of Clinical Microbiology, v. 41, p. 623-626, 2003.

LINDSAY, D. S.; BLAGBURN, B. L.; ERNEST, J. A. Experimental Cryptosporidium infections in chickens. Journal of Parasitology, v. 73, p. 242-244, 1987.

O'DONOGHUE, P. J. Cryptosporidium and cryptosporidiosis in man and animals. International Journal of Parasitology, v. 25, p. 139-195, 1995.

POWER, M.; RYAN, U. Cryptosporidium macropodum n. sp. (Apicomplexa: Cryptosporidiidae) from eastern grey kangaroos Macropus giganteus. Journal of Parasitology, v. 11, n. 1, p. 1.114-1.117, 2008.

RYAN, U. M.; POWER, M.; XIAO, L. Cryptosporidium fayeri n. sp. (Apicomplexa: Cryptosporidiidae) from the Red Kangaroo 
(Macropus rufus). The Journal of Eukaryotic Microbiology, v. 55, n. 1, p. 22-26, 2008.

SMITH, H. V.; CACCIÒ, S. M; COOK, N.; NICHOLS, R. A.; TAIT, A. Cryptosporidium and Giardia as foodborne zoonoses. Veterinary Parasitology, v. 149, p. 29-40, 2007.

SUNNOTEL, O.; LOWERY, C. J.; MOORE, J. E.; DOOLEY, J. S. G.; XIAO, L.; MILLAR, B. C. Cryptosporidium. Letters in Applied Microbiology, v. 43, p. 7-16, 2006.

THOMPSON, R. C. A.; CHALMERS, R. M. Cryptosporidium: from molecules to disease. Trends in Parasitology, v. 18, p. 98-100, 2002.

TZIPORI, S. Cryptosporidiosis in animals and humans. Microbiological Reviews, v. 47, p. 84-96, 1983.
UPTON, S. J.; MCALLISTER, C. T; FREED, P. S. Cryptosporidium spp in wild and captive reptiles. Journal of Wildlife Diseases, v. 25, p. 20-30, 1989.

WIDMER, G.; LIN, L.; KAPUR, V.; FENG, X.; ABRAHAMSEN, M. S. Genomics and genetics of Cryptosporidium parvum: the key to understanding cryptosporidiosis. Microbes and Infection, v. 4, p. 1.081-1.090, 2002.

XIAO, L.; FAYER, R.; RYAN, U., UPTON, S. J. Cryptosporidium taxonomy: recent advances and implications for public health. Clinical Microbiology Reviews, v. 17, n. 1, p. 72-97, 2004.

Protocolado em: 3 jan. 2008. Aceito em: $1^{\circ}$ out. 2010. 